\title{
Solubility of Anthracene in Ternary Dibutyl Ether + Alcohol + Heptane Solvent Mixtures at 298.15 K
}

\author{
Karen J . Pribyla and William E. Acree, J r.* \\ Department of Chemistry, University of North Texas, Denton, Texas 76203-5070
}

\begin{abstract}
Experimental solubilities are reported for anthracene dissolved in ternary dibutyl ether +1 -propanol + heptane, dibutyl ether + 2-propanol + heptane, dibutyl ether + 1-butanol + heptane, dibutyl ether + 2-butanol + heptane, and dibutyl ether + 2-methyl-1-propanol + heptane solvent mixtures at $25^{\circ} \mathrm{C}$ and atmospheric pressure. Nineteen compositions were studied for each of the five solvent systems. Results of these measurements are used to test the predictive ability of the ternary solvent form of the combined NIMS/Redlich-Kister equation. Computations showed that the model predicted the observed solubility behavior to within an overall average absolute deviation of about $1.5 \%$, which is comparable to the experimental uncertainty of $\pm 1.5 \%$.
\end{abstract}

\section{Introduction}

Solid-liquid equilibrium data of organic nonelectrolyte systems are becoming increasingly important in the petroleum industry, particularly in light of present trends toward heavier feedstocks and the known carcinogenicity/ mutagenicity of many of the larger polycyclic aromatic compounds. Solubility data for a number of polycyclic aromatic hydrocarbons (i.e., anthracene and pyrene) and heteroatom polynuclear aromatics (i.e., carbazole, di benzothiophene, and xanthene) have been published in the recent chemical literature. For a listing of references see Acree (1994, 1995a,b). Despite efforts by experimentalists and scientific organizations, both in terms of new experimental measurements and critically evaluated data compilations, there still exist numerous systems for which solubility data are not readily available.

In the present study anthracene solubilities have been measured in five ternary dibutyl ether + alcohol + heptane systems at $25{ }^{\circ} \mathrm{C}$. Nineteen ternary compositions were studied for each of the five systems. Results of these measurements are used to test the predictive ability of expressions based upon the general mixing model used in deriving the combined NIBS/Redlich-Kister equation.

\section{Experimental Methods}

Anthracene (Acros, 99.9+\%) was recrystallized three times from 2-propanone. 1-Propanol (Aldrich, 99+\%, anhydrous), 2-propanol (Aldrich, 99+\%, anhydrous), 1-butanol (Aldrich, HPLC, 99.8+\%), 2-butanol (Aldrich, 99+\%, anhydrous), 2-methyl-1-propanol (Aldrich, 99.5\%, anhydrous), heptane (Aldrich, HPLC, 99+\%), and dibutyl ether (Aldrich, 99.3\%, anhydrous) were stored over molecular sieves and distilled shortly before use. Gas chromatographic analysis showed solvent purities to be $99.7 \mathrm{~mol} \%$ or better. Ternary solvent mixtures were prepared by mass so that compositions could be calculated to 0.0001 mole fraction. The methods of sample equilibration and spectrophoto-

* To whom correspondence should be addressed. e-mail: acree@ unt.edu. Fax: (940) 565-4318. metric analysis are discussed in an earlier paper (Powell et al., 1997). Experimental anthracene solubilities in the five dibutyl ether + alcohol + heptane solvent mixtures are listed in Table 1. Numerical values represent the average of between four and eight independent determinations, with the measured values being reproducible to within $\pm 1.5 \%$.

\section{Results and Discussion}

Acree and co-workers (Acree, 1992; Acree and Zvaigzne, 1991; Acree et al., 1991) suggested the combined NIBS/ Redlich-Kister equation for the mathematical representation of isothermal solubility data in binary solvent systems

$$
\ln x_{A}^{s a t}=x_{B}^{\circ} \ln \left(x_{A}^{s a t}\right)_{B}+x_{C}^{\circ} \ln \left(x_{A}^{s a t}\right)_{C}+x_{B}^{\circ} x_{C}^{\circ} \sum_{i=0}^{n} S_{i}\left(x_{B}^{\circ}-x_{C}^{\circ}\right)^{i}
$$

where $x_{B}^{\circ}$ and $x_{C}^{\circ}$ refer to the initial mole fraction composition of the binary solvent calculated as if the solute were not present and $\left(x_{A}^{\text {sat }}\right)_{i}$ denotes the measured solute solubility in pure solvent $i$. The various $S_{i}$ curve-fit parameters can be evaluated with a least-squares analysis. For a ternary solvent system, the mathematical representation takes the form

$$
\begin{array}{r}
\ln x_{A}^{\text {sat }}=x_{B}^{\circ} \ln \left(x_{A}^{\text {sat }}\right)_{B}+x_{C}^{\circ} \ln \left(x_{A}^{\text {sat }}\right)_{C}+x_{D}^{\circ} \ln \left(x_{A}^{\text {sat }}\right)_{D}+ \\
x_{B}^{\circ} x_{C}^{\circ} \sum_{i=0}^{r} S_{i, B C}\left(x_{B}^{\circ}-x_{C}^{\circ}\right)^{i}+x_{B}^{\circ} x_{D}^{\circ} \sum_{j=0}^{s} S_{j, B D}\left(x_{B}^{\circ}-x_{D}^{\circ}\right)^{j}+ \\
x_{C}^{\circ} x_{D}^{\circ} \sum_{k=0}^{t} S_{k, C D}\left(x_{C}^{\circ}-x_{D}^{\circ}\right)^{k}
\end{array}
$$

Recent studies have shown that eq 2 provides reasonably accurate predictions for anthracene solubilities in ternary two alkane + alcohol (Deng and Acree, 1998a; Deng et al., 1999a) and alkane + two alcohol (Deng and Acree, 1998b; Deng et al., 1998 and 1999b) solvent mixtures. Such systems exhibit fairly large deviations from solution ideality arising from the self-association of each alcohol cosol- 
1260 J ournal of Chemical and Engineering Data, Vol. 44, No. 6, 1999

Table 1. Experimental Mole Fraction Solubilities of Anthracene $\left(x_{A}^{\text {sat }}\right)$ in Ternary Dibutyl Ether (B) + Alcohol $(C)+$ Heptane (D) Solvent Mixtures at $298.15 \mathrm{~K}$

\begin{tabular}{|c|c|c|c|c|c|c|c|c|}
\hline$x_{B}^{\circ}$ & $x_{C}^{\circ}$ & $x_{A}^{\text {sat }}$ & $x_{B}^{\circ}$ & $x_{C}^{\circ}$ & $x_{A}^{\text {sat }}$ & $x_{B}^{\circ}$ & $x_{C}^{\circ}$ & $x_{A}^{\text {sat }}$ \\
\hline \multicolumn{9}{|c|}{ Dibutyl Ether (B) + 1-Propanol (C) + Heptane (D) } \\
\hline 0.2156 & 0.4883 & 0.001771 & 0.0582 & 0.6332 & 0.001247 & 0.0728 & 0.3039 & 0.001604 \\
\hline 0.0833 & 0.8087 & 0.001042 & 0.2409 & 0.6702 & 0.001635 & 0.0598 & 0.7858 & 0.001030 \\
\hline 0.1736 & 0.3808 & 0.001777 & 0.3160 & 0.5829 & 0.001957 & 0.1475 & 0.1787 & 0.001796 \\
\hline 0.1508 & 0.6460 & 0.001441 & 0.1106 & 0.2440 & 0.001706 & 0.0682 & 0.5334 & 0.001382 \\
\hline 0.5668 & 0.2090 & 0.002802 & 0.5496 & 0.2765 & 0.002789 & 0.2989 & 0.1932 & 0.002128 \\
\hline 0.5307 & 0.3533 & 0.002694 & 0.3491 & 0.4060 & 0.002158 & 0.3801 & 0.1870 & 0.002310 \\
\hline 0.1062 & 0.8154 & 0.001073 & & & & & & \\
\hline \multicolumn{9}{|c|}{ Dibutyl Ether (B) + 2-Propanol (C) + Heptane (D) } \\
\hline 0.2275 & 0.5065 & 0.001686 & 0.0664 & 0.6514 & 0.001119 & 0.0802 & 0.3299 & 0.001554 \\
\hline 0.0843 & 0.8172 & 0.000873 & 0.2460 & 0.6726 & 0.001467 & 0.0585 & 0.8147 & 0.000829 \\
\hline 0.1837 & 0.4095 & 0.001705 & 0.3283 & 0.5865 & 0.001801 & 0.1689 & 0.1899 & 0.001821 \\
\hline 0.1528 & 0.6687 & 0.001280 & 0.1244 & 0.2662 & 0.001679 & 0.0735 & 0.5603 & 0.001256 \\
\hline 0.5867 & 0.2124 & 0.002844 & 0.5708 & 0.2795 & 0.002763 & 0.3320 & 0.1972 & 0.002228 \\
\hline 0.5445 & 0.3604 & 0.002651 & 0.3601 & 0.4187 & 0.002126 & 0.4149 & 0.2018 & 0.002380 \\
\hline 0.1119 & 0.8176 & 0.000927 & & & & & & \\
\hline \multicolumn{9}{|c|}{ Dibutyl Ether (B) + 1-Butanol (C) + Heptane (D) } \\
\hline 0.2513 & 0.4580 & 0.001993 & 0.0757 & 0.6158 & 0.001447 & 0.0808 & 0.2986 & 0.001703 \\
\hline 0.0964 & 0.7913 & 0.001248 & 0.2762 & 0.6356 & 0.001916 & 0.0656 & 0.7851 & 0.001209 \\
\hline 0.1983 & 0.3638 & 0.001895 & 0.3584 & 0.5467 & 0.002223 & 0.1690 & 0.1717 & 0.001931 \\
\hline 0.1682 & 0.6280 & 0.001664 & 0.1297 & 0.2351 & 0.001796 & 0.0802 & 0.5185 & 0.001556 \\
\hline 0.6137 & 0.1748 & 0.003024 & 0.5962 & 0.2489 & 0.002910 & 0.3376 & 0.1781 & 0.002338 \\
\hline 0.5788 & 0.3198 & 0.002861 & 0.3900 & 0.3754 & 0.002425 & 0.4234 & 0.1801 & 0.002544 \\
\hline 0.1250 & 0.7955 & 0.001330 & & & & & & \\
\hline \multicolumn{9}{|c|}{ Dibutyl Ether (B) + 2-Butanol (C) + Heptane (D) } \\
\hline 0.2524 & 0.4595 & 0.001880 & 0.0746 & 0.6175 & 0.001308 & 0.0826 & 0.2921 & 0.001659 \\
\hline 0.1268 & 0.7278 & 0.001278 & 0.2759 & 0.6369 & 0.001726 & 0.0652 & 0.7881 & 0.001060 \\
\hline 0.1964 & 0.3626 & 0.001866 & 0.3640 & 0.5399 & 0.002047 & 0.1714 & 0.1637 & 0.001892 \\
\hline 0.1781 & 0.6210 & 0.001542 & 0.1280 & 0.2295 & 0.001772 & 0.0784 & 0.5205 & 0.001460 \\
\hline 0.6113 & 0.1772 & 0.002909 & 0.5913 & 0.2523 & 0.002858 & 0.3387 & 0.1759 & 0.002233 \\
\hline 0.5762 & 0.3165 & 0.002762 & 0.3941 & 0.3715 & 0.002319 & 0.4265 & 0.1711 & 0.002420 \\
\hline 0.1269 & 0.7901 & 0.001171 & & & & & & \\
\hline \multicolumn{9}{|c|}{ Dibutyl Ether (B) + 2-Methyl-1-Propanol (C) + Heptane (D) } \\
\hline 0.2541 & 0.4532 & 0.001815 & 0.0753 & 0.6137 & 0.001175 & 0.0833 & 0.2919 & 0.001562 \\
\hline 0.0958 & 0.7912 & 0.000968 & 0.2807 & 0.6333 & 0.001616 & 0.0679 & 0.7838 & 0.000917 \\
\hline 0.1957 & 0.3630 & 0.001784 & 0.3619 & 0.5407 & 0.002003 & 0.1731 & 0.1666 & 0.001888 \\
\hline 0.1773 & 0.6224 & 0.001382 & 0.1286 & 0.2319 & 0.001752 & 0.0798 & 0.5198 & 0.001310 \\
\hline 0.6149 & 0.1748 & 0.002910 & 0.5963 & 0.2453 & 0.002832 & 0.3384 & 0.1793 & 0.002223 \\
\hline 0.5803 & 0.3132 & 0.002725 & 0.3924 & 0.3733 & 0.002213 & 0.4235 & 0.1839 & 0.002461 \\
\hline 01278 & 38 & 0.00102 & & & & & & \\
\hline
\end{tabular}

vent and, in mixtures containing two alcohol cosolvents, from the formation of heterogeneous hydrogen-bonded chains between dissimilar alcohol molecules.

The predictive ability of eq 2 is summarized in Table 3 for anthracene dissolved in the five dibutyl ether + alcohol + heptane systems. Unlike the case of the ternary two alkane + alcohol and alkane + two alcohol solvent mixtures studied previously, hydrogen-bond formation is terminated each time that an alcohol molecule hydrogen bonds with dibutyl ether. Published papers (Zvaigzne et al., 1993 and 1994; Zvaigzne and Acree, 1994; Powell and Acree, 1995; Acree et al., 1994) have reported the calculated $S_{i}$ parameters for anthracene dissolved in ten of the eleven subbinary solvent systems, as well as the measured mole fraction solubilities in 1-propanol $\left(x_{A}^{\text {sat }}=0.000591\right)$, 2-propanol $\left(x_{A}^{\text {sat }}=0.000411\right)$, 1-butanol $\left(x_{A}^{\text {sat }}=0.000801\right)$, 2-butanol $\left(x_{A}^{\text {sat }}=0.000585\right), 2$-methyl-1-propanol $\left(x_{A}^{\text {sat }}=\right.$ $0.000470)$, heptane $\left(x_{A}^{\text {sat }}=0.001571\right)$ and dibutyl ether $\left(x_{A}^{\text {sat }}=0.003615\right)$. Solubility data for the eleventh binary solvent system were reported several years prior to the devel opment of the combined NIBS/Redlich-Kister equation. We have determined the numerical values of the $S_{i}$ parameters for the binary dibutyl ether + heptane solvent system by curve fitting the experimental anthracene mole fraction solubility data of Marthandan and Acree (1987) in accordance with eq 1 . Numerical values of the $S_{i}$ parameters have been tabulated in Table 2 for convenience. Examination of the numerical entries in Table 3 reveals that eq 2 predicts the solubility of anthracene to within an overall average absolute deviation of $1.5 \%$, which is
Table 2. Combined NIBS/Redlich-Kister Parameters Calculated from Anthracene Solubilities in the Sub-binary Solvent Systems

\begin{tabular}{cr}
\hline solvent (B) + solvent (C) & \multicolumn{1}{c}{$\mathrm{S}^{\mathrm{a}}$} \\
\hline 2-methyl-1-propanol (B) + heptane (C) & 1.284 \\
& 0.301 \\
2-propanol (B) + heptane (C) & 0.266 \\
& 1.491 \\
2-butanol (B) + heptane (C) & 0.587 \\
1-propanol (B) + heptane (C) & 0.566 \\
& 1.225 \\
1-butanol (B) + heptane (C) & 0.292 \\
& 1.098 \\
& 0.106 \\
dibutyl ether (B) + 1-propanol (C) & 0.324 \\
& 0.723 \\
dibutyl ether (B) + 2-propanol (C) & -0.004 \\
& 0.201 \\
& 2.167 \\
dibutyl ether (B) + 1-butanol (C) & -0.931 \\
& 0.891 \\
& 2.588 \\
dibutyl ether (B) + 2-butanol (C) & -1.235 \\
& 0.866 \\
dibutyl ether (B) + 2-methyl-1-propanol (C) & 1.736 \\
& 0.488 \\
dibutyl ether (B) + heptane (C) & 0.574 \\
& 2.109 \\
& -0.849 \\
& 0.726 \\
& 2.231 \\
& -0.932 \\
& 0.927 \\
& 0.220
\end{tabular}

a Combined NIBS/Redlich-Kister curve-fit parameters are ordered as $\mathrm{S}_{0}, \mathrm{~S}_{1}$, and $\mathrm{S}_{2}$. 
Table 3. Summarized Comparison between Observed Anthracene Solubilities in Ternary Dibutyl Ether + Alcohol + Heptane Solvent Mixtures and Predicted Values Based upon the Combined NIMS/Redlich-Kister Equation 2

\begin{tabular}{lcc}
\hline \multicolumn{1}{c}{ ternary solvent mixture } & $\begin{array}{c}\% \\
\text { dev }^{\mathrm{a}}\end{array}$ & $\begin{array}{c}\% \text { max. } \\
\text { dev }^{\mathrm{b}}\end{array}$ \\
\hline dibutyl ether (B) + 1-propanol (C) + heptane (D) & 1.78 & 4.55 \\
dibutyl ether (B) + 2-propanol (C) + heptane (D) & 1.37 & 2.90 \\
dibutyl ether (B) + 1-butanol (C) + heptane (D) & 1.70 & 3.49 \\
dibutyl ether (B) + 2-butanol (C) + heptane (D) & 1.22 & 3.21 \\
dibutyl ether (B) + 2-methyl-1-propanol (C) & 1.65 & 3.32 \\
+ heptane (D) & &
\end{tabular}

a Deviation $(\%)=(100 / N) \sum\left|\left[\left(x_{A}^{\text {sat }}\right)^{\text {calc }}-\left(x_{A}^{\text {sat }}\right)^{\exp }\right] /\left(x_{A}^{\text {sat }}\right)^{\exp }\right|$, where $\mathrm{N}$ corresponds to the number of data points for each ternary system. In the present study, solubilities were determined at 19 different ternary solvent compositions. ${ }^{b}$ Maximum deviation (\%) $=100\left|\left[\left(x_{\mathrm{A}}^{\text {sat }}\right)^{\text {calc }}-\left(x_{\mathrm{A}}^{\text {sat }}\right) \exp \right] /\left(x_{\mathrm{A}}^{\text {sat }}\right) \exp \right|$.

comparable to the experimental uncertainty of $\pm 1.5 \%$. For the five systems studied, eq 2 was found to provide very accurate predictions of the observed solubility behavior.

\section{Literature Cited}

Acree, W. E., J r. Mathematical Representation of Thermodynamic Properties. Part 2. Derivation of the Combined Nearly I deal Binary Solvent (NIBS)/Redlich-Kister Mathematical Representation from a Two-Body and Three-Body Interactional Mixing Model. Thermochim. Acta 1992, 198, 71-79.

Acree, W. E., Ir. Polycydlic Aromatic Hydrocarbons in Pureand Binary Solvents; Volume 54 in IUPAC Solubility Data Series; Oxford University Press: Oxford, U.K., 1994.

Acree, W. E., J r. Polycyclic Aromatic Hydrocarbons: Binary Nonaqueous Systems: Part 1 (Solutes A-E); Volume 58 in IUPAC Solubility Data Series; Oxford University Press: Oxford, U.K., 1995a.

Acree, W. E., J r. Polycyclic Aromatic Hydrocarbons: Binary Nonaque ous Systems: Part 2 (Solutes F-Z); Volume 59 in IUPAC Solubility Data Series; Oxford University Press: Oxford, U.K., 1995b.

Acree, W. E., J r.; Zvaigzne, A. I. Thermodynamic Properties of Nonelectrolyte Solutions. Part 4. Estimation and Mathematical Representation of Solute Activity Coefficients and Solubilities in Binary Solvents Using the NIBS and Modified Wilson Equations. Thermochim. Acta 1991, 178, 151-167.

Acree, W. E., J r.; McCargar, J. W.; Zvaigzne, A. I.; Teng, I.-L. Mathematical Representation of Thermodynamic Properties. Car- bazole Solubilities in Binary Alkane + Dibutyl Ether and Alkane + Tetrahydropyran Solvent Mixtures. Phys. Chem. Liq. 1991, 23, 27-35.

Acree, W. E., J r.; Zvaigzne, A. I.; Tucker, S. A. Thermochemical Investigations of Hydrogen-Bonded Solutions. Development of a Predictive Equation for the Solubility of Anthracene in Binary Hydrocarbon + Alcohol Mixtures Based U pon Mobile Order Theory. Fluid Phase Equilib. 1994, 92, 233-253.

Deng, T.; Acree, W. E., J r. Solubility of Anthracene in Ternary Propanol $+2,2,4-$ Trimethylpentane + Cyclohexane and Butanol + 2,2,4Trimethylpentane + Cyclohexane Solvent Mixtures. J . Chem. Eng. Data 1998a, 43, 1059-1061.

Deng, T.; Acree, W. E., J r. Solubility of Anthracene in Ternary Propanol + Butanol + Cyclohexane Solvent Mixtures. J . Chem. Eng. Data 1998b, 43, 1062-1064.

Deng, T.; Childress, S. D.; De Fina, K. M.; Sharp, T. L.; Acree, W. E., J r. Solubility of Anthracene in Ternary Propanol + Butanol + 2,2,4 Trimethylpentane Solvent Mixtures. J . Chem. Eng. Data 1998, 43, 1065-1067.

Deng, T.; Hernández, C. E.; Roy, L. E.; Acree, W. E., J r. Solubility of Anthracene in Ternary (Propanol + Heptane + Cyclohexane) and (Butanol + Heptane + Cyclohexane) Solvent Mixtures. J . Chem. Thermodyn. 1999a, 31, 205-210.

Deng, T.; Childress, S. D.; De Fina, K. M.; Acree, W. E., J r. Solubility of Anthracene in Ternary Propanol + Butanol + Heptane Solvent Mixtures. Chem. Eng. Commun. 1999b, 172, 217-224.

Marthandan, M. V.; Acree, W. E., J r. Solubility of Anthracene in Binary Solvent Mixtures Containing Dibutyl Ether. J . Chem. Eng. Data 1987, 32, 301-303.

Powell, J. R.; Acree, W. E., J r. Solubility of Anthracene in Binary Alcohol + Dibutyl Ether Solvent Mixtures. J . Chem. Eng. Data 1995, 40, 914-916.

Powell, J . R.; Coym, K. S.; Acree, W. E., J r. Solubility of Anthracene in Binary Alcohol + 2-Methoxyethyl Ether Solvent Mixtures. J . Chem. Eng. Data 1997, 42, 395-397.

Zvaigzne, A. I.; Acree, W. E., J r. Solubility of Anthracene in Binary Alkane + 2-Butanol Solvent Mixtures. J. Chem. Eng. Data 1994 39, 114-116.

Zvaigzne, A. I.; Teng, I.-L.; Martinez, E.; Trejo, J .; Acree, W. E., J r. Solubility of Anthracene in Binary Alkane + 1-Propanol and Alkane + 1-Butanol Solvent Mixtures. J. Chem. Eng. Data 1993, 38, 389392.

Zvaigzne, A. I.; Wolfe, J .; Acree, W. E., J r. Solubility of Anthracene in Binary Alkane + 2-M ethyl-1-propanol Solvent Mixtures. J . Chem. Eng. Data 1994, 39, 541-543.

Received for review May 10, 1999. Accepted J uly 23, 1999.

J E990130R 